\title{
« Notre peuple a perdu le sens de la danse honnête ». Danses africaines, catégories légales et (re)définitions européennes de l'obscénité dans le Congo colonial
}

\author{
Amandine Lauro
}

Amandine Lauro : Amandine Lauro est chercheure postdoctorale au FNRS à l'Université libre de Bruxelles, professeure invitée à l'Université Saint-Louis et membre du CRHIDI depuis 2012. Depuis plusieurs années, ses recherches s'articulent autour de l'histoire de la colonisation en Afrique centrale et de l'histoire du genre et de la sexualité en situation coloniale. Elle a publié sur la sexualité interraciale et la prostitution coloniale, le genre et le droit colonial et plus récemment sur les politiques de maintien de l'ordre et de surveillance des frontières raciales au Congo Belge.

DOI: $10.25518 / 1370-2262.246$

\section{Résumé :}

Dès le début du $\mathrm{XX}^{\mathrm{e}}$ siècle, les autorités coloniales du Congo Belge se sont inquiétées de la régulation de danses congolaises qualifiées d'obscènes, présentées comme de véritables menaces pour l'ordre moral (voire public) de la jeune colonie. Cet article a précisément pour but d'interroger ces évolutions discursives et normatives ainsi que les projets d'encadrement légal auxquelles elles ont donné lieu. Il analyse les manières dont l'évolution des catégories coloniales du moral et de l'immoral qui s'opère au cours de l'entre-deux-guerres se répercute dans les objets mouvants de la catégorie légale des danses obscènes et des festivités qui y sont associées. Il souligne que le renouvellement des inquiétudes européennes autour de ces pratiques récréatives témoigne d'anxiétés plus larges liées aux limites du contrôle colonial des espaces urbains et des lieux et pratiques de sociabilité (nocturnes) qui s'y développent. Ces questions offrent un terrain particulièrement propice pour explorer les limites de la surveillance coloniale de la fête et de ses espaces dans l'Afrique centrale de la première moitié du XX $\mathrm{XX}^{\mathrm{e}}$ siècle ainsi que les manières dont le droit colonial a été mobilisé, en des termes et selon des usages évolutifs et flexibles, pour tenter d'y remédier.

Mots-clés : Colonie, Congo, Danse, Régulation sociale

\section{Abstract :}

From the early $20^{\text {th }}$ century, colonial authorities in the Belgian Congo became worried about Congolese dances described as « obscene » and presented as threats for the moral (and public) order of the colony. This article aims precisely at interrogating these discursive and normative evolutions as well as the projects of legal regulation born of the anxieties. It analyses the evolutions of colonial definitions of « morality » through the interwar period and the ways in which they reflected in the moving boundaries of the official category of « obscene dances ». It underlines that the renewal of European concerns about these recreational practices testifies to the existence of larger anxieties linked to the limits of colonial control in urban spaces and in their developing places of (nightlife) sociabilities. These questions offer a particularly interesting base to explore the limits of colonial surveillance of spaces of feasts and celebrations in Central Africa in the first half of the 20th century and to study how colonial law has been used, in evolutive and flexible ways, to try to counter these limits. 
« Notre peuple a perdu le sens de la danse honnête ». Danses africaines, caté...

Keywords : Congo, Dance, Public order, Social regulation

\section{Introduction}

En 1937, Joseph Van Wing, missionnaire catholique influent officiant au Congo Belge depuis plusieurs années $\underline{1}$, publie un article intitulé Les danses Bakongo dans larevue coloniale belge la plus importante de l'époque2. Auteur de plusieurs ouvrages ethnographiques sur les populations de la région, Van Wing livre cette fois une réflexion à vocation plus critique que descriptive sur les évolutions récentes de ces danses : sa sentence "notre peuple a perdu le sens de la danse honnête $» \underline{3}$ résonne en effet comme un constat implacable de la (supposée) perversion croissante de ces danses sous les assauts d'une modernité corruptrice. Si ce point de vue est alors loin d'être exceptionnel dans le monde colonial belgo-congolais (y compris en dehors des rangs religieux qui ne sont pas les seuls, en contexte colonial, à investir le terrain de l'ordre moralú), trente ans plus tôt, plus d'un missionnaire aurait pourtant été surpris d'apprendre que les danses congolaises avaient un jour été « honnêtes ».

$\mathrm{Au}$ tournant du siècle, la plupart des récits occidentaux sur l'Afrique centrale comportent une scène de danse. Passage obligé parmi les «scènes et types » qui font le piquant de la littérature exotique, les descriptions des « danses indigènes » sont l'occasion d'illustrer le caractère " primitif » des Africains en ce qu'elles permettent d'évoquer non seulement l'exubérance et l'omniprésence de leurs pratiques festives, mais aussi un «éternel africain » où la sexualité joue un rôle de premier plan. Inséparables du double processus d'exotisation et d'érotisation du corps africain et de ses usages qui a accompagné le mouvement colonial des $\mathrm{XIX}^{\mathrm{e}}$ et $\mathrm{XX}^{\mathrm{e}}$ siècles $\underline{5}$, ces représentations constituent des témoignages intéressants de la place stratégique de la sexualité dans la construction des catégories raciales et de la mission civilisatrice $\underline{6}$. À ce titre, on ne s'étonnera pas qu'elles aient nourri non seulement des imaginaires raciaux et culturels mais aussi des politiques de répression, orchestrées dans le contexte congolais par les autorités européennes dès le début du $\mathrm{XX}^{\mathrm{e}}$ siècle, visant à rendre illégales les danses dites « obscènes » ainsi désignées comme de véritables menaces pour l'ordre moral de la jeune colonie belge.

C'est précisément à l'articulation de ces projets de régulation légale et des évolutions discursives et normatives qui les ont accompagnés que cet article s'intéresse. À la différence d'autres étudesz, il s'agit ici d'interroger les manières dont les évolutions des catégories coloniales du moral et de l'immoral et le glissement progressif du «moralement incorrect » des espaces «traditionnels » et coutumiers vers les espaces de la «modernité » et extracoutumiers qui s'opère dans l'entre-deuxguerres se répercutent dans les objets mouvants de la catégorie légale des "danses obscènes » et des festivités qui y sont associées. En effet, alors que les premières dispositions en la matière visaient à réguler une série de performances associées à la «tradition » et aux milieux ruraux, les nouvelles stratégies répressives qui émergent dans l'entre-deux-guerres s'articulent plutôt autour des nouvelles cultures de la fête qui se développent dans les centres urbains $\underline{8}$ et qui sont marquées par des performances musicales et chorégraphiques d'une nature inédite posant de nouveaux problèmes - et de nouveaux défis - aux autorités. Comme dans bien d'autres contextes, l'instauration de nouvelles normes en matière de danse ne vise pas seulement le contrôle des corps, mais aussi la définition de « what acceptable behaviour is in relation to others, and (...) how moralities ought to be embodied $\underline{9}$ ». Si la danse est un langage, ici révélateur, comme d'autres travaux l'ont montré, des nouvelles identités urbaines et des relations sociales et de pouvoir qui 
s'y jouent10, elle est aussi un moment festif qui se développe en dehors de l'initiative coloniale et où s'expriment certaines frustrations et/ou contestations 11 . Les inquiétudes de l'administration européenne autour de ces nouvelles pratiques récréatives témoignent en fait d'anxiétés plus larges liées aux limites du contrôle colonial des espaces urbains et des lieux et pratiques de sociabilité (nocturnes) qui s'y développent. Elles constituent donc un terrain particulièrement propice pour explorer les limites de la surveillance coloniale de la fête et de ses espaces dans l'Afrique centrale de la première moitié du $\mathrm{XX}^{\mathrm{e}}$ siècle ainsi que les manières dont le droit colonial a été mobilisé, en des termes et selon des usages évolutifs et flexibles, pour tenter d'y remédier.

\section{Regards européens sur le corps africain en mouvement}

Du journal d'exploration au récit de voyage, du grand reportage journalistique au film de propagande, les scènes de danseconstituent souvent un passage obligé pour les auteurs européens lorsqu'il s'agit d'évoquer l'Afrique centrale. Véritable exercice de style, les descriptions de ces danses dites «primitives » figurent en bonne place parmi les «traditions curieuses » des colonisés (polygamie, tatouages, etc.)12. Elles présentent néanmoins une spécificité : elles ont presque toujours un caractère sexuel. En décalage avec «les canons de la motricité européenne » $\underline{13}$, les danses africaines font encore souvent figure, dans les premières années du $\mathrm{XX}^{\mathrm{e}}$ siècle, de témoignages de l'irrémédiable lascivité des populations qui s'y livrent, suggérant l'idée d'une sexualité moins contrainte socialement. Le stéréotype primitiviste du « rythme dans la peau » participe d'ailleurs du succès des « revues nègres » dans l'Europe de l'entre-deux-guerres14 : les « danses africaines » telles qu'elles sont alors imaginées et mises en scène sont l'occasion pour les spectateurs blancs de « retrouver l'innocence primordiale de l'humanité, recouvrer des instincts bridés et dévoyés par la civilisation industrielle, se livrer en toute candeur aux plaisirs et aux joies de la vie naturelle »15, et d'entrevoir « les fureurs de l'Eros africain » 16 par la même occasion.

«Grossière pantomime érotique » 17 pour les uns, «dreadful bacchanalian exhibition » 18 ou série de «poses provocantes » $\underline{19}$ pour les autres, les danses congolaises sont, sous le regard des premiers colonisateurs, de parfaites démonstrations de l'immoralité supposée régner chez les natifs. Celle-ci fait partie, on le sait, du répertoire d'arguments mobilisés à l'appui de la définition de l'infériorité des colonisés et de la construction des hiérarchies raciales en situation coloniale. Les articulations de la race, de la sexualité et du genre sont d'ailleurs très visibles dans les récits de danses. D'une part, ces derniers mettent généralement en scène des femmes (ou plutôt des corps de femmes) dont les performances semblent s'adresser personnellement au spectateur blanc (masculin) telle « une véritable invitation sexuelle proposée à l'étranger. » 20 D'autre part, décrites de manière quasi systématique comme sensuelles et caractérisées par des mouvements du bassin et du plexus dont les observateurs européens font une interprétation strictement sexuelle, les scènes de danse servent aussi de prétextes à des digressions sur la nature hyper(hétéro)sexuelle des Africain(e)s : « Cette danse donne lieu à des gestes obscènes ; il en est de même pour toutes les danses pratiquées par les indigènes hors de la présence de l'Administration. Tout comme le latin en ses mots brave l'honnêteté, le noir trouve parfaitement naturel de singer des gestes qu'il accomplît et qu'il voit accomplir journellement sous ses yeux par les animaux domestiques qui divaguent dans les villages. Le geste de la reproduction est pour le noir la seule raison d'être ; c'est le geste qui perpétue la race; il ne peut donc pas être ignoble » $\underline{1} 1$, souligne ainsi un administrateur dans un rapport à vocation « ethnographique » des années 1930.

C'est que les danses congolaises ne sont pas seulement obscènes en elles-mêmes; elles le sont 
« Notre peuple a perdu le sens de la danse honnête ». Danses africaines, caté...

aussi en raison des «débordements » qui les suivent et auxquels elles semblent inévitablement mener. On pourrait ainsi multiplier les citations décrivant les «danses nocturnes dégénérant régulièrement en effroyables orgies, et scènes de soulographie, dont les descriptions les plus réalistes ne donneraient qu'une faible idée à qui n'a jamais vu les noirs dans leur milieu naturel »22, dont le spécialiste de la littérature coloniale française Alain Ruscio a fourni un parfait résumé (s'appliquant tout à fait à la prose colonisatrice belge) : «Rituel immuable : mâles et femelles mêlés s'approchent, se frottent, s'excitent ; le désir croit ; la musique assourdissante, toujours sur le même rythme, ajoute à l'excitation ; l'atmosphère s'électrise. Toute pudeur, si toutefois il en eut, disparaît. Chaque mâle se rue sur la première femelle qui passe à sa portée. Les accouplements commencent. Tout se passe au vu et au su de tous, en plein air, dans la nature (comme il se doit). Les singes du zoo de Vincennes se gênent-ils ?23”»

Dans ce contexte, on ne s'étonnera d'ailleurs pas de ce que les missionnaires aient été parmi les pourfendeurs les plus acharnés des «danses traditionnelles » congolaises. Ils se révèlent ainsi particulièrement enclins à critiquer l'obscénité de ces performances en raison et de la nature de leurs chorégraphies, et de leurs fonctions de préliminaires : de nombreuses danses « en soi ne sont pas inconvenantes, mais aboutissent souvent à la licence, à cause des circonstances qui d'ordinaire les accompagnent: chants, excès de boisson, exaltation malsaine produite par la répétition frénétique d'un même rythme, penchant pervers des danseurs, tambours, obscurité voulue pour l'exécution, etc. (...) Les circonstances qui accompagnent ces danses enivrent et affolent les sens, affaiblissent la volonté, émoussent la conscience. Ces danses dangereuses sont condamnées par la morale parce qu'elles constituent des occasions prochaines de péché24»". Sans surprise, ils seront aussi les premiers à souhaiter inscrire leur éradication sur la liste des priorités civilisationnelles de l'autorité coloniale, comme l'a bien souligné Yolanda Covington-Ward로.

\section{Haro sur les danses traditionnelles}

Ces condamnations des danses congolaises ne sont pas seulement discursives; elles sont aussi légales. Dès 1900, les autorités de l'État Indépendant du Congo (EIC) (propriété personnelle du Roi des Belges Léopold II depuis 1885), s'étaient attaquées à la régulation des danses via une mesure de police destinée à octroyer aux autorités administratives la possibilité de réglementer certaines «danses indigènes » exécutées sur la voie publique. Il s'agissait cependant d'une disposition générale avant tout motivée par l'importance, «dans un intérêt de sécurité et de tranquillité publique, de restreindre dans certaines limites les danses indigènes et d'empêcher d'autre part qu'elles ne constituent une entrave à la libre circulation sur les voies publiques 26 ». Elle ne visait donc pas spécifiquement le caractère potentiellement licencieux des danses, à l'image d'un régime peu préoccupé par l'ordre moral de la société coloniale et par les responsabilités civilisatrices qu'il s'était assigné. Il faut d'ailleurs noter que jusqu'à la fin de la période coloniale, certaines danses seront l'objet de préoccupations (et d'interdictions) en raison de la menace qu'elles représenteraient pour l'ordre public pour des motifs politiques (danses liées aux mouvements du Kimbanguisme ou du Kitawala par exemple) 27 ou économiques («Il est pratiquement impossible aux danseurs de travailler normalement le lendemain (...). »)228.

Il faut donc attendre 1912 (soit quatre ans à peine après la "reprise », qui a vu l'EIC devenir le Congo Belge, colonie nationale) pour que l'administration coloniale s'attaque explicitement à ce qui va désormais être officiellement qualifié de « danses obscènes ». Cette démarche passe dans un premier temps par une nouvelle interprétation des dispositions de 1900, qu'il s'agit de mobiliser 
contre les «danses coutumières d'un caractère nettement lascif ou obscène » : « De la part des autorités territoriales, chargées d'être les agents de la civilisation auprès des populations indigènes, tolérer ces pratiques et ne pas les réprimer pourrait être à juste titre considéré par elles comme une marque d'approbation »29. Il est d'ailleurs précisé que seules les danses ne représentant pas de danger ni pour l'ordre public, ni pour l'ordre moral, peuvent être réglementées; les danses obscènes doivent quant à elles être purement et simplement interdites. L'année suivante, une nouvelle circulaire est édictée, preuve que la question des « danses obscènes » agite l'administration - et probablement aussi les autorités missionnaires : elle vient rappeler aux fonctionnaires que leur devoir est de s'opposer à ces pratiques « qui constituent un obstacle permanent à tous les efforts civilisateurs et qui s'opposent à l'accession des populations indigènes au niveau intellectuel et moral auquel nous avons entrepris de les élever ». Le texte invite également les agents coloniaux à faire preuve d'autorité en les incitant à sévir en cas de récidive via l'établissement de procèsverbaux pour outrage aux mœurs $\underline{30}$. Enfin, moins de deux ans plus tard, les danses sont à nouveau l'objet de prescriptions officielles, même s'il s'agit cette fois de mettre en garde les fonctionnaires contre les excès d'une application trop rigoureuse des instructions précédentes et de leur rappeler que seules les « pratiques à tendance nettement obscènes » doivent être prohibées $\underline{31}$.

Ces mesures participent clairement de la dynamique de réforme morale qui est celle d'une partie du monde colonial belge dans la foulée de la «reprise ». Marquée par la volonté de promouvoir une colonie exemplaire, moralement irréprochable, et de faire oublier les entorses faites à la «mission civilisatrice » sous le régime léopoldien, le climat de cette période de transition est marqué par l'expression de préoccupations pour le «bien-être » des populations «indigènes » et pour la suppression de coutumes «barbares », notamment dans les domaines du mariage et de la sexualité. Pour les autorités, il s'agit d'affirmer la priorité qu'occupe le relèvement moral et avec lui les objectifs civilisateurs parmi les politiques de la «nouvelle » Belgique coloniale - à moindres frais : il est en effet a priori plus facile de s'engager sur le terrain des «moeurs » que sur celui de l'économie politique et des cadres structurels de l'exploitation coloniale du Congo tels qu'ils avaient prévalu jusque-là (travail et recrutement forcé, violence contre les populations, etc.).

On ne dispose malheureusement que de peu d'informations sur la mise en œuvre de ces instructions. Il semble toutefois clair qu'elles n'aient pas été appliquées massivement. Dans les années 1920, le monde missionnaire continue à critiquer «les soulographies publiques, avec danses obscènes et immoralité indescriptible », appelant de ses vœux l'établissement de "sanctions très sévères » en la matière. $\underline{32}$ Face à ce qu'il considère comme un certain immobilisme de la part des autorités coloniales, il va jusqu'à prendre dans certaines régions des initiatives répressives (à caractère religieux - exclusion de la communion par exemple $\underline{33}$ - ou plus général - amendes et confiscation d'instruments à percussion). Illustrations des micro-conflits qui ont émaillé le quotidien de la gestion coloniale «trinitaire » du Congo, ces initiatives empiètent sur les prérogatives régaliennes de l'administration, au grand dam de celle-ci34. Dans la région du Bas-Congo, étudiée par Y. Covington-Ward, les missionnaires - et en particulier les Protestants - se révèlent particulièrement pro-actifs en la matière $\underline{35}$. Périodiquement, des fonctionnaires font eux aussi état du peu de cas qui est fait de ces possibilités réglementaires et des lacunes de la surveillance exercée par leurs subordonnés $\underline{36}$ alors que pourtant « les danses obscènes se multiplient partout, d'après les missionnaires catholiques » $\underline{37}$. Il faut dire aussi que dans ce domaine, la tâche des fonctionnaires n'était pas des plus simples. Outre les mécontentements que risquaient de générer d'éventuelles interdictions, on peut aisément imaginer les difficultés éprouvées par l'administration pour «débusquer » ces danses, les populations congolaises n'ignorant probablement rien de la 
« Notre peuple a perdu le sens de la danse honnête ». Danses africaines, caté...

réprobation dont elles étaient l'objet. Comme le rappelait un administrateur, l'outrage aux mœurs est difficile à établir puisque ce type de danse «ne se pratique [pas] en présence ou à proximité d'un représentant de l'autorité. Il devrait donc se prouver par des témoignages ; c'est assez dire qu'on n’y parviendra guère » $\underline{38}$. Les missionnaires ne disent pas autre chose, estimant que «le Noir, sachant les danses obscènes prohibées, évitera en présence des blancs tout ce qui peut les choquer et provoquer leur intervention » $\underline{39}$. Pour éviter ce travers, les missionnaires ont d'ailleurs une solution toute prête : utiliser les services de chrétiens réputés pour leur fidélité et leur droiture (des catéchistes par exemple) qui, introduits dans le milieu " indigène », sont à même de renseigner aux missionnaires quelles danses sont obscènes (ou non) et qui les pratique.

Il est vrai que souvent, les administrateurs territoriaux semblent moins prompts que les missionnaires à « découvrir » des danses obscènes dans les régions où ils exercent, en tous cas dans les années 1930. Lors des enquêtes administratives menées au cours de cette décennie, beaucoup estiment que ces danses sont tombées en désuétude ou que, si elles existent encore, ce n'est que ponctuellement. L'impression qui domine est qu'à quelques exceptions près, les agents de l'État colonial ne prêtent pas un fol intérêt à cette question, ce qui en soi témoigne de certaines évolutions. Certes, nul ne cherche à nier que certaines danses présentent un caractère « immoral », mais la circonspection est désormais de mise, "parce que nous n'avons pas le droit de prendre des mesures en cette matière, et que nous ne sommes pas des censeurs de mours (...)40 ». En fait, les «danses obscènes » n'apparaissent précisément plus que comme des mœurs à censer, et non plus comme les menaces pour l'ordre moral qu'elles représentaient quelques années auparavant. En la matière, c'est d'ailleurs que vient désormais le danger : des danses dites « modernes » et des dancings urbains qui constituent leurs espaces privilégiés.

\section{Les danses urbaines sous surveillance}

\section{Danser en ville au Congo Belge}

Étroitement liées au développement des villes et aux innovations qui ont marqué leur paysage social, culturel et récréatif, les danses urbaines émergent au Congo à partir des années 1920 en dehors de tout contrôle colonial. Les travaux de C.D. Gondola ont bien montré comment ces nouvelles formes musicales et chorégraphiques ont constitué, pour les populations congolaises, un des seuls espaces de sociabilité, de récréation et de création libre (ou presque) de toute intervention coloniale. Pourtant (ou peut-être à cause de cela), comme on va le voir, le développement de cette nouvelle culture musicale, et principalement des danses qui y sont associées, n'est pas sans inquiéter les autorités.

Dès 1923, le chroniqueur belge Chalux mentionne l'existence de plusieurs salles de danse réservées aux Africains à Léopoldville⒈ Ce témoignage corrobore les hypothèses de Phyllis Martin, qui estime quant à elle que les dancings ont fait leur apparition dans les deux Congo dans les années 1920, voire un peu avant42. Si dans un premier temps, ces danses de couple nouvelles semblent amuser les Européens qui viennent même y assister, en spectateurs $\underline{43}$, elles vont rapidement les inquiéter. Les premiers à se préoccuper des conséquences néfastes de ces manifestations sont, sans surprise, les missionnaires. Le développement de styles de danse inspirés à la fois des danses latino-américaines et des danses européennes, et notamment du style «maringa », les inquiète particulièrement. 
En quoi consistent ces danses précisément ? Leur émergence est indissociable du développement de nouveaux styles musicaux dans les villes du Congo et de leur syncrétisme ethnique. Progressivement, une nouvelle culture musicale va naître de la rencontre entre univers (et héritages) musicaux variés que permet la ville. Elle s'articule aussi autour de la prolétarisation des travailleurs africains, pour qui la musique populaire va constituer un terrain d'expression culturelle et de « socialité créative »44 à l'abri du contrôle colonial. Le syncrétisme des musiques populaires urbaines a d'ailleurs été favorisé par le rôle nouveau qu'acquiert la performance musicale et ce qu'elle englobe (musique en elle-même, textes des chants, danses) en milieu urbain. Même s'il faut se garder du cliché selon lequel les danses « traditionnelles » africaines seraient systématiquement des manifestations spiritualo-religieuses $\underline{45}$, il est clair que la musique urbaine « joue de plus en plus un rôle ludique » 46 par rapport à celui qu'elle exerçait en milieu rural, en même temps qu'elle témoigne de la nouvelle donne coloniale, de ses contraintes, et des nouveaux rapports sociaux et de pouvoir qui se mettent en place dans les centres $\underline{4}$.

Diverses influences ont nourri le développement de la musique congolaise urbaine et, par extension, des danses qui l'ont accompagnée. Outre les apports des musiques «traditionnelles » qui constituent sans doute les sources les plus fécondes de la musique populaire, il faut mentionner d'une part la rencontre de la musique et de la chanson européenne $\underline{48}$ et d'autre part l'apport des chants scouts, fanfares militaires et autres chorales religieuses respectivement développées par les autorités de la Force Publique et de nombreuses missions religieuses. 49 La source d'influence principale de la musique congolaise est cependant sans conteste à rechercher du côté des rythmes latino-cubano-américains et du highlife des côtes occidentales de l'Afrique, introduits par les coastmen, ces travailleurs venus d'Afrique de l'ouest. 50 Ces influences se matérialisent par une familiarité nouvelle (et une maîtrise croissante) à l'égard de nouvelles sonorités, de nouveaux instruments ; elles font aussi émerger de nouvelles pratiques matérielles d'écoute (disques vinyles, etc.) et de performance. Ainsi, si dans l'entre-deux-guerres, le paysage musical congolais est surtout caractérisé par des musiciens travaillant en solo, exerçant leurs talents à l'occasion de fêtes ou de cérémonies diverses, les débuts de la Deuxième Guerre mondiale marquent l'émergence des orchestres qui allaient par la suite se multiplier et investir de manière croissante les bars et... les dancings.

C'est que ces nouvelles musiques s'accompagnent de nouvelles danses. La maringa et la rumba furent des innovations tant au point de vue musical stricto sensu qu'au point de vue chorégraphique. Le style «maringa » est particulièrement intéressant car il a connu un développement spectaculaire dans l'entre-deux-guerres, bien avant que la rumba n'ait le succès que l'on connaît. Originaire, selon toute vraisemblance, de l'Afrique occidentale française, le style maringa n'avait pas d'identité ethnique claire, ce qui favorisa la rapidité et l'étendue de sa diffusion. Les morceaux joués étaient des combinaisons de mélodies «traditionnelles » avec des rythmes empruntés à la musique latinocubaine (polkas, fandangos) et à d'autres styles européens (marches militaires, hymnes religieux, airs français). Les airs maringa symbolisent donc parfaitement la confluence de ces influences qui, comme le souligne Gary Steward, « recalled the turn-of-the-century New Orleans and the mixing of African, Afro-Spanish, and French musical elements into jazz » $\underline{1}$. D'un point de vue chorégraphique, les danses maringa sont des danses de couple, caractérisées par des mouvements de hanche qui amènent les danseurs à placer le poids de leur corps alternativement sur une jambe et puis l'autre, un peu à la manière de la rumba (à qui elle a donc largement préparé le terrain) 52 . Leur diffusion rapide (dans la plupart des centres du Bas-Congo notamment) est inséparable de la création de multiples dancings et bars-dancings qui sont pour beaucoup, on le verra, dans 
« Notre peuple a perdu le sens de la danse honnête ». Danses africaines, caté...

les critiques dont ces danses nouvelles feront l'objet53. Des dancings qui verront aussi éclore le succès d'autres danses dès les années 1930 (fox-trots, tangos, biguines, etc.), notamment chez les élites urbaines émergentes, $\underline{54}$ avant que la rumba, musique d'origine afro-cubaine que le Congo exportera dans toute l'Afrique subsaharienne et qui « was born to be danced » $\underline{5}$, ne vienne tout balayer sur son passage $\underline{56}$.

\section{Des nouveaux usages de la catégorie « danse obscène »}

Dès leur développement, ces nouvelles danses vont faire l'objet de critiques en raison de leur supposée obscénité, dans des termes qui rappellent ceux qui étaient utilisés au début du siècle pour évoquer les danses «traditionnelles » des populations congolaises. Les nouveaux rapports au corps, à la mixité de genre, à la sexualité et à l'espace public que ces performances impliquent soulèvent en effet de nombreuses inquiétudes.

Pour bon nombre de leurs détracteurs européens, l'élément le plus problématique de ces danses urbaines réside dans leur pratique en couple : les danseurs, un homme et une femme, évoluent ensemble et se tiennent enlacés, un élément qui, pour beaucoup, rompt avec la tradition africaine et favorise les rapprochements suspects. Le modus operandi de ces danses rappelle inévitablement aux Européens leurs propres danses (valses, fox-trotts, etc) qui se performent également en couple et qui constituent, dans le Congo colonial d'alors, un loisir de choix. Pourtant, peu semblent se réjouir que les Africains se soient mis à danser des "danses de civilisés ». Aux yeux des Occidentaux, les Congolais pervertissent en fait les danses européennes, les dénaturent en leur conférant une obscénité qu'elles ne possédaient pas à l'origine. Comme le résumait un magistrat, « cette innovation [est] fort regrettable; elle l'est pour la morale, car les danses où un couple se tient entrelacé, inconnues de la coutume indigène, ont pour les noirs une portée licencieuse qu'elles n'ont pas chez nous $״ \underline{57}$. Beaucoup prennent - à tort - les danses maringa pour des imitations de danses européennes que les Africains auraient « sexualisées » par des mouvements de hanche notamment - alors qu'il s'agissait, comme on l'a vu, non pas d'imitations « ratées » de danses européennes mais de performances innovantes largement inconnues sur le continent européen.

Cette application à « singer les blancs » est, en particulier, attribuée à l'élite émergente (clercs, travailleurs qualifiés, domestiques, etc.) des villes du Congo de l'entre-deux-guerres. C'est elle qui est accusée de propager la maringa et de faire des danses européennes «des évolutions licencieuses $" \underline{58}$, elle aussi qui cherche à «s'amuser comme les blancs (évidemment pas comme la généralité des blancs font, qui s'amusent honnêtement, mais comme certains font, et dont ils savent tous les procédés). (...) Dans ces réunions, il y a bon nombre de jeunes filles qui assistent. On y mange beaucoup, on y boit beaucoup et on y danse! Et quelles danses ! (...) Ces danses et beuveries sont une source de disputes, de bagarres et de discorde dans les familles $\underline{59}$ ». Certes, des historiens ont effectivement montré que ce sont les élites africaines qui ont les premières dansé la maringa, la biguine et la rumba dans les dancings, tandis que la masse des urbains continuait de pratiquer essentiellement des danses de plein air plus «traditionnelles » sur une base plus ethnique. À certains égards, l'émergence de ces nouvelles danses fut même étroitement liée au développement d'une identité de groupe parmi les élites urbaines du Congo colonial 60 (en même temps qu'elle a constitué un moyen et un vecteur d'affirmation individuelle au sein de ce groupe61), même s'il n'existait pas de barrière étanche entre les danseurs «traditionnels » et les danseurs «modernes » $\underline{62}$. Cette coexistence se révèle d'ailleurs problématique du point de vue des autorités coloniales. Non seulement les urbains sont accusés de pervertir une série de danses 
a priori «saines » (« certaines danses qui, à l'origine, n'avaient aucun caractère licencieux ou pornographiques le sont devenus à Léopoldville par suite des figures introduites par les danseurs, habituellement les simulacres de l'amour »63), mais ils se voient aussi reprocher, par leur mobilité, de contaminer les milieux ruraux, au grand dam des missionnaires : « La question est tout autre lorsqu'il s'agit des danses modernes introduites dans les villages de brousse par des Européens ou par certains travailleurs revenant des centres extra-coutumiers. Toutes ces danses sont exécutées à la fois par hommes et femmes, qui forment souvent des couples au grand scandale des anciens. Aussi devra-t-on en général les défendre aux chrétiens. Il est intéressant de noter à ce sujet que les chefs de villages sont unanimes à condamner ces danses; mais ils sont impuissants à les empêcher n'ayant plus pour cela l'autorité nécessaire $» \underline{64}$.

La ville pervertit donc tout. Les inquiétudes des autorités coloniales autour des danses urbaines illustrent la manière dont le développement du discours colonisateur sur la sexualité « dangereuse » et plus généralement sur l'immoralité des villes africaines trahit des anxiétés plus générales au sujet des populations urbaines et des limites des stratégies d'encadrement déployées par le pouvoir colonial. L'évocation, dans la citation qui précède, de l'impuissance des chefs traditionnels à faire changer leurs subordonnés de conduite en est une parfaite métaphore. Les discours à la fois critiques et normatifs sur l'obscénité des danses urbaines reflètent donc des préoccupations plus larges, et il n'est pas étonnant que l'on y retrouve des critiques liées à l'immoralité, à l'alcool et la prostitution, fléaux associés à l'urbanité. En effet, c'est aussi tout le contexte qui entoure les danses qui est mis en cause, ces manifestations "devant dégénérer inévitablement dans ce milieu si disparate d'individus de toutes origines en des scènes immorales et perturbatrices $\underline{65}$ » : « la danse à l'intérieur des habitations (...) favorise l'entrée des femmes dans ces maisons, dans lesquelles salles de dégustation de boissons fermentées et de danse ne font qu'une, qui est située à proximité des chambres à coucher, où les femmes sont attirées par plusieurs moyens, ce qui lorsque cela arrive à la connaissance des maris trompés, provoque des palabres sans fin $\underline{66}$ ». La perte de religiosité est également pointée du doigt comme une conséquence de la propagation de ces nouvelles danses : certains pensent même que «les danses préoccupent à ce point la majorité de leurs fidèles [des missions] que ceux-ci abandonnent la pratique des devoirs religieux67 ». Pour d'autres, tel l'Evêque du Katanga Jean-Félix De Hemptinne, c'est même une question de statistique : «Depuis cette nouvelle réglementation [autorisations d'ouverture de salles de danse], la fréquentation des catéchismes a diminué de $30 \%$. Il en va de même pour la fréquentation des sacrements. Cette incompatibilité entre les divertissements admis et exploités et la pratique religieuse représente le verdict de la conscience indigène 68 ».

Cette évolution rejoint (en même temps qu'elle s'explique par) des mutations plus larges, inscrites à la fois dans un contexte socio-politique particulier et dans le pragmatisme de la gestion coloniale. Après les années de tumulte qui ont entouré la reprise de 1908, l'administration belge se retrouve face aux défis que pose la gestion quotidienne du Congo et l'intensification de son exploitation économique. Les débats autour des orientations qu'il convient de donner à la «politique indigène » sont nombreux et sortent renforcés d'une Première Guerre mondiale qui donne le temps à l'administration de constater la complexité des pratiques congolaises et les limites des interventions européennes. Le régime colonial belge évolue alors vers des modes d'administration plus indirects, s'appuyant sur les structures de l'autorité coutumière. Cette évolution se traduit également par une revalorisation de l'encadrement social, politique et moral «traditionnel » de manière générale. Simultanément à la construction de la ville comme foyer de perversion s'opère donc une construction de l'espace rural comme refuge (ou plutôt bastion) de l'harmonie et de la discipline 
« Notre peuple a perdu le sens de la danse honnête ». Danses africaines, caté...

sociale et familiale de la «communauté » africaine «authentique » régie par les règles et les institutions « coutumières ». La catégorie de l'immoralité change alors d'objet et surtout d'espace. Elle se déplace vers les sexualités urbaines présentées comme sources de désordre et conséquences de la déliquescence, en ville, du «sain » contrôle coutumier, une évolution qui renvoie aussi au difficile problème politique de la coexistence d'un cadre administratif et bureaucratique qui mobilise largement l'organisation coutumière avec les impératifs du développement économique de la colonie et de sa nécessaire urbanisation.

Que ceux qui sont désignés comme les principales cibles en la matière soient précisément d'une part les «Évolués », cette élite urbaine émergente dont on connaît les craintes qu'elle a pu inspirer à l'administration coloniale, et d'autre part les Coastmen, ces travailleurs et entrepreneurs étrangers venus d'Afrique de l'Ouest69 eux aussi soupçonnés de velléités contestataires, est, au-delà de la pratique effective de ces danses, en soi significatif. De même, les préoccupations tatillonnes des autorités urbaines pour les dancings, ces lieux clos qui offrent la possibilité d'un entre-soi soustrait au regard public, cristallisent des anxiétés plus larges liées au contrôle des espaces de sociabilité urbains qui échappent à la mainmise coloniale. Pourquoi vouloir danser à l'abri des regards si ce n'est pour cacher quelque chose ? L'obsession de certains représentants du pouvoir colonial pour l'éclairage, le nombre de fenêtres et l'ouverture des portes d'entrée de ces dancings est à cet égard révélatrice. À Élisabethville, le curé de la paroisse ira même jusqu'à solliciter auprès des autorités de la ville l'installation de l'électricité à l'intérieur et aux abords des salles de danse, avec succès $\underline{70}$. Certains missionnaires se sont d'ailleurs lancé dans de véritables croisades contre les salles de danse, allant jusqu'à « se mettre à l'affût, le soir, dans les environs de la salle de danse (...) la plus proche de la Mission, pour saisir tous les incidents, si anodins soient-ils, qui doivent servir à prouver les méfaits dont la danse est responsable ", quitte à faire passer une panne de courant de deux minutes pour une plongée volontaire de la salle dans une obscurité propice aux rapprochements. 71 Certes, en métropole aussi, les dancings (ces « serres chaudes de la corruption ») sont l'objet de nombreuses suspicions 72 , mais ces inquiétudes ont en contexte colonial des accents particuliers.

\section{Un dispositif réglementaire pour les danses urbaines?}

La surveillance des salles de danse n'est pas seulement le fait de quelques missionnaires zélés. L'administration coloniale a elle aussi cherché à traduire en dispositions réglementaires ses inquiétudes. Cette démarche implique nécessairement l'élaboration de mesures inédites, car les législations sur les « danses indigènes » du début du siècle ne s'attaquaient qu'aux danses exécutées sur la voie publique. De manière intéressante, ces premiers règlements avaient précisément pour but d'éviter que certaines danses «obscènes » ne se déroulent en public, aux yeux de tous; la publicité constituait donc un élément essentiel du délit. Quelques années plus tard, alors que la publicité des danses est devenue un gage de leur correction et leur caractère privé un motif d'inquiétude, ce type de dispositions pose problème. Cette impasse juridique constitue d'ailleurs un bon exemple de l'évolution des préoccupations coloniales en matière de " danses obscènes ». Un fonctionnaire de l'administration le faisait bien remarquer en 1936 : « les danses indigènes, comme toute matière intéressant l'ordre et la tranquillité, peuvent faire l'objet d'une réglementation. (...) Les premières [les danses ayant lieu dans un local privé] ne peuvent être réglementées, chacun étant maître chez soi, sous réserve du respect des droits d'autrui et de la loi73 ». Quelques années plus tôt, le parquet d'Élisabethville avait été confronté à un problème similaire, obligé de reconnaître son impuissance malgré la multiplication des plaintes : "Étant donné les conditions dans lesquelles ces danses se pratiquent en locaux fermés, l'arrêté du 17 juillet 1910 ne peut 
recevoir application $\gg$ 74 . Les stratégies réglementaires mises en place à partir des années 1930 se concentreront donc non plus sur les danses elles-mêmes, mais bien sur les espaces matériels où elles se déroulent. Une série d'ordonnances provinciales se succèdent en effet dès le début des années 193075. Elles visent spécifiquement les danses en milieu urbain et consistent en mesures de portée assez générale : il s'agit surtout d'établir un cadre légal de base autour des « établissements de danse » (ségrégation raciale, conditions d'ouverture et d'octroi de licence, etc.)76, qui sera confirmé dans les années 194077.

C'est cependant au niveau local que sont prises l'essentiel des dispositions visant spécifiquement à réguler le développement des dancings en lien avec leur caractère licencieux supposé. En raison précisément de ce champ d'application restreint, il est difficile d'en dresser un tableau exhaustif (ces mesures ne sont pour la plupart pas publiées au Bulletin Officiel). D'archives administratives disparates, il ressort néanmoins que dans la capitale congolaise, les salles de danses sont par exemple purement et simplement interdites par les autorités de la ville en 1930 et 193178. Au milieu des années 1940, ce sont une série de danses qui y sont prohibées en raison de leur caractère « obscène 79 ». L’autre grande métropole du Congo Belge, Élisabethville, se préoccupe du sujet dès la deuxième moitié des années 1920. Administrateurs et magistrats cherchent alors à élaborer un règlement permettant de limiter autant que possible leur développement et surtout leur immoralité supposée80. Ce n'est pourtant qu'en 1931 que la question prend un tour plus décisif, avec un règlement, cette fois concrétisé, qui n’autorise les danses que les samedis, dimanches et jours fériés entre 13 et 21 heures; les seules danses autorisées «sont les danses coutumières n'offrant aucun danger pour l'ordre ou la tranquillité publique, ou ne revêtant aucun caractère lascif ou obscène 81 ». Cela revient à prohiber les danses « modernes » qui ne pourront être autorisées que de manière exceptionnelle et à la condition qu'elles prennent place dans un local « ouvert » de la cité, pas trop rapproché des débits de boissons82. Deux ans plus tard, alors que la ville avait entre-temps autorisé l'ouverture de dancings, ceux-ci voient leur licence supprimée au profit d'une salle unique et officielle : ainsi, « les indigènes se mélangeront; la surveillance sera plus facile et les accrocs à la morale moins aisés et moins nombreux 83 ». Dès lors, témoignage de la volonté des autorités coloniales de garder la main sur les espaces de festivités africains, seul un bâtiment communal (la " maison du centre ») sera autorisé à accueillir des séances de danses en salle, après que le nombre de dancings "privés » ait été progressivement réduit au cours de l'année 1933, grâce à une surveillance tatillonne organisée et ouvertement destinée à prendre les tenanciers en défaut (« ce nombre devra être réduit petit à petit en appliquant strictement la réglementation en vigueur et en éliminant les salles qui ne rempliraient pas toutes les conditions voulues au point de vue hygiène, éclairage, etc... ou dont les tenanciers toléreraient des actes ou attitudes contraires aux bonnes mœurs 84 »). Ce sera chose faite en 1934, bien que ces mesures n'aient évidemment pas obtenu, à long terme, le succès escompté85.

\section{Conclusion : Dirty dancing, paniques morales et ordre colonial}

Ces réglementations parfois très minutieuses et les visées normatives (voire répressives) qui les portent ne semblent en effet avoir eu qu'une portée limitée, à Élisabethville comme ailleurs. De la fin de la Deuxième Guerre mondiale à l'Indépendance, la danse - et ses lieux : dancings, bars, ... ne fera que se développer dans les villes du Congo Belge. Elle participe d'une culture de la fête à la fois innovante et subversive qui « sert de tribune » à la « sociabilité urbaine » comme à « l'esprit d'indiscipline 86 » des populations et qui ouvre des espaces de discussion et de performance des identités, des rôles sociaux et enfin du politique dans les villes congolaises. Dans le chef des 
« Notre peuple a perdu le sens de la danse honnête ». Danses africaines, caté...

autorités coloniales, les inquiétudes exprimées face au développement des nouvelles formes de danses urbaines et leur qualification d'obscénité trahissent, on l'a vu, des anxiétés plus vastes autour des pratiques sociales urbaines et des limites du contrôle colonial. Les danses obscènes font ainsi partie d'un répertoire de paniques morales autour des modernités urbaines et des désordres familiaux, sexuels et de genre représentatifs de la menace qui pèse sur l'ordre "imaginé » des communautés africaines «traditionnelles », un répertoire qui justifie une surveillance qui n'est pas toujours proportionnelle à l'enjeu des danses en tant que telles. En dépit de leurs effets limités, les dispositions réglementaires sur les danses obscènes constituent un observatoire intéressant des évolutions des définitions de la menace en situation coloniale et des priorités à établir en la matière, y compris en matière de divertissement. Il serait à cet égard intéressant de s'interroger sur la contribution des populations congolaises et de leurs élites au renouvellement de ces anxiétés étroitement liées aux définitions d'une respectabilité aux contours évolutifs, aux définitions du caractère décent - ou non - de certaines danses $\underline{87}$ et à leurs recompositions postcoloniales $\underline{88}$, au prisme du genre notamment.

Le contenu mouvant de la qualification de « danses obscènes » se répercute d'ailleurs également sur les nouvelles appréciations dont les danses «traditionnelles » sont l'objet à partir des années 1930. Illustrant tout à la fois « les pièges de la ville et la reconquête de la brousse89 », le glissement progressif du « moralement incorrect » vers les espaces urbains de la « modernité » se traduit ainsi par une revalorisation des vertus des danses «traditionnelles ». Celles-ci apparaissent désormais bien moins comme des manifestations primitives incompatibles avec la civilisation que comme de saines expressions récréatives authentiquement «indigènes ». Même les missionnaires admettent désormais, à l'exemple du Père Van Wing, que « la danse constitue pour les Bantous l'exercice récréatif par excellence $\underline{90}$ ». Les danses «traditionnelles » seraient donc par essence « honnêtes »: là où cela n'est pas le cas, il ne fait aucun doute que l'introduction d'éléments «modernes » est à blâmer : il ne peut s'agir que de «la conséquence d'une certaine européanisation. Il est donc inexact de dire que les danses licencieuses sont inhérentes à l'état moral des Africains $\underline{91}$ ». Cette logique prévaut jusque dans l'encadrement qui est proposé aux quelques Congolais autorisés à s'aventurer en métropole : l'institution destinée à accueillir les marins de passage dans le port d'Anvers prévoit ainsi lors de sa création des animations musicales avec percussions traditionnelles qui « permettront les trémoussements des danses de leurs villages » afin de réduire la tentation des « cabarets des environs immédiats » et de leurs jazz-bands92. Le double contexte global, d'une part du développement de nouvelles pratiques en matière de loisirs collectifs « sains » de plein air, axés sur l'épanouissement du corps et les bienfaits du sport (la danse est aussi un exercice physique), et d'autre part de patrimonialisation des arts africains (la danse est aussi un art), n'est sans doute pas non plus étranger à cette évolution, dans des proportions qui restent à déterminer.

En dépit de ces évolutions, on peut toutefois s'interroger sur la portée sexuelle que conserveront les danses africaines aux yeux des Européens. Dans le cinéma colonial par exemple, les scènes de danses restent «sexuellement significatives » $\underline{93}$ bien au-delà de l'entre-deux-guerres. Il faut dire que leur appel visuel et leur performativité permettent une représentation très incarnée de l'Afrique «traditionnelle » dont les spectateurs européens demeurent friands. Les danses semblent toujours permettre un accès direct «à toutes les émotions qu'éveille le contact avec la vie équatoriale primitive 94 » et à une Afrique éternelle et « authentique ». Cette mise en spectacle et en folklore des danses «traditionnelles » congolaises, et leur omniprésence dans les films comme dans la promotion du tourisme ne fait cependant pas que des heureux. Dans les années 1950, dans un contexte où la Belgique s'efforce de démontrer l'effectivité de son programme civilisateur 
de modernisation et de développement du Congo95, l'administration coloniale s'agace de la multiplication des reportages qui « contribuent à fausser dangereusement les idées des lecteurs ou des spectateurs par ailleurs peu avertis du degré d'évolution des populations congolaises et des résultats de notre action civilisatrice en Afrique. Pour l'opinion internationale, il vaudrait mieux exposer objectivement nos réalisations sociales et économiques plutôt que présenter comme actualités des survivances de pratiques arriérées et barbares souvent provoquées artificiellement pour satisfaire certains reporters $\underline{96}$ ». Même si le sujet reste à développer, il est évident que les Congolais ne furent bien sûr jamais dupes de ces mises en scène ni des usages tout à la fois érotisants et primitivistes que les Européens ont pu en faire - bien au-delà de la période coloniale d'ailleurs. En 1955 déjà, dans le Congo modèle de Bwana Kitoko, ce célèbre film réalisé à l'occasion de la visite du Roi Baudouin dans la colonie, José Lobeya, rédacteur en chef de l'hebdomadaire catholique La Croix du Congo, ne se privait pas de pointer du doigt la longue et éculée scène de danse mettant en scène des corps congolais, artefacts plus que sujets de l'action, " dans leur état le plus primitif », au détriment de l'élite congolaise et de ses interactions avec le Roi, apparemment bien moins photogéniques $\underline{97}$.

\section{Notes}

1 J. Denis, Wing (Van), Joseph, dans Biographie Belge d'Outre-Mer, Bruxelles, ARSOM, vol. 8, 1998, col. 461-470.

2 J. VAN Wing, Les danses Bakongo, dans Congo. Revue Générale de la Colonie belge, juillet 1937, t. 2 .

3 Idem, p. 128.

4 Pour un aperçu des spécificités du contexte belgo-congolais, voir A. LAURO, Les politiques du mariage et de la sexualité au Congo Belge (1908-1945). Genre, race, sexualité et pouvoir colonial, Thèse de doctorat en histoire, Université Libre de Bruxelles, 2009, dont le présent article dérive (p. 347-387). Voir également N.R. HunT, Noise over Camouflaged Polygamy, Colonial Morality Taxation, and a Woman-Naming Crisis in Belgian Africa, dans Journal of African History, vol. 32, 1991.

$\underline{5}$ Bien que l'articulation des représentations des danses "primitives » et de l'altérité culturelle et raciale ait une plus longue histoire comme en atteste A. ARCANGELI, Dancing Savages : Stereotypes and Cultural Encounters across the Atlantic in the Age of European Expansion, dans Exploring Cultural History. Essays in Honour of Peter Burke, éd. M. CALARESU et al., New York, Ashgate, 2010.

6 Parmi une vaste littérature consacrée à ces sujets, voir notamment Domesticating the Empire : Race, gender and family life in French and Dutch Colonialism, éd. J. CLANCY-SMITH ET et F. GoudA, Charlottesville, University Press of Virginia, 1998 ; le désormais classique A-L. STOLER, Carnal Knowledge and Imperial Power. Race and the Intimate in Colonial Rule, Berkeley-Los Angeles, University of California Press, 2002 ; ou encore la synthèse Gender and Empire, éd. P. LEvinE, Oxford, Oxford University Press, 2004. Sur le définition et la régulation de l' "obscénité » en situation coloniale, voir aussi D. HEATH, Purifying Empire : Obscenity and the Politics of Moral Regulation in Britain, India and Australia, Cambridge, Cambridge University Press, 2010.

$\underline{7}$ Nous pensons ici essentiellement au travail de Y. COVInGTON-WARD, Threatening Gestures, 
« Notre peuple a perdu le sens de la danse honnête ». Danses africaines, caté...

Immoral Bodies : The Intersection of Church, State and Kongo Performance in the Belgian Congo, dans Missions, States and European Expansion in Africa, éd. C. KoRICH et R. NJOKU, New York, Routledge, 2007, centré sur la région du Bas-Congo et dont nous n'avons eu connaissance que récemment.

$\underline{8}$ Sur le cas particulier du Congo, voir l'incontournable C.D. Gondola, 'Bisengo y a la joie'. Fête, sociabilité et politique dans les capitales congolaises, dans Fêtes urbaines en Afrique. Espaces, identités, pouvoirs, éd. O. Goerg, Paris, Karthala, 1999. Sur les cultures populaires de la fête dans l'Afrique coloniale, voir le volume Fêtes urbaines en Afrique. Espaces, identités, pouvoirs... et le classique D. Coplan, In Township Tonight! Musique et théâtre dans les villes noires d'Afriques du Sud, Paris, Karthala, 1992.

9 H. Neveu Kringelbach, Movement, Morality and Self-Fashioning in Urban Senegal, New York/ Oxford, Oxford University Press, 2013, p. 7.

10 Dans le contexte congolais, voir B. JewsIEwICKI, Danse et construction de l'identité de la personne moderne en Afrique centrale, dans Danse : Langage propre et métissage culturel, éd. C. PontBriand, Montréal, Parachute, 2002 ; ID., Dance : The Passion to Be Oneself in Dialogue with Others, dans Remembering Africa, éd. E. MudimBe-Boy, Portsmouth, Heinemann, 2002 ; Musique urbaine au Katanga. De Malaika à Santu Kimbangu, éd. B. JeWsieWiCKI , Paris, L'Harmattan, 2003. Voir également C.D. Gondola, Popular Music, Urban Society, and Changing Gender Relations in Kinshasa, Zaire, dans Gendered Encounters : Challenging Cultural Boundaries and Social Hierarchies in Africa, éd. M. Grosz-NGATÉ et O.H. KoKOLE, New York, Routledge, 1996 ; Idem, Ô, Kisasa makambo! Métamorphoses et représentations urbaines de Kinshasa à travers le discours musical des années 1950-1960, dans Le Mouvement Social, vol. 204, 2003, t. 3. Dans une perspective plus contemporaine, voir aussi B. WhITE, Rumba Rules. The Politics of Dance in Mobutu's Zaïre, Durham, Duke University Press, 2008. En dehors du Congo, voir entre autres les pionniers J. CLYDE MITCHELL, The Kalela dance : Aspects of social relationships among urban Africans in Northern Rhodesia, Manchester, Manchester University Press, 1956 et T. RANGER, Dance and Society in Eastern Africa, Londres, Heineman, 1975. Plus récemment, voir entre autres M. SuRIANo, Making the Modern : Contestations over Muziki wa Dansi in Tanganyika, ca. 1945-1961, dans African Studies, vol. 70, 2011, t. 3.

11 C.D. Gondola, 'Bisengo y a la joie'... ; C.D. Gondola, Villes miroirs : migrations et identités urbaines à Kinshasa et Brazzaville, 1930-1970, Paris, L'Harmattan, 1996, p. 231-278. Voir aussi P. MARTIN, Leisure and Society in Colonial Brazzaville, Cambridge, Cambridge University Press, 1995.

12 C'est notamment frappant dans les supports de la propagande congolaise, L. VINTS, Kongo made in Belgium. Beeld van een kolonie in film en propaganda, Louvain, Kritak, 1984, p. 42. Sur les représentations des corps colonisés dans la culture coloniale belge, voir B. HENRIET, Regard blanc, corps sauvage. La représentation filmée des corps coutumiers au Congo belge (1924-1939). Imaginaire, genre, ethnicité. Mémoire de master en Histoire, Université Libre de Bruxelles, 2012.

13 Zoos Humains, de la vénus hottentote aux Reality Shows, éds N. BANCEL, P. BlanchaRd, G. Boetsch, E. Deroo et S. Lemaire, Paris, La Découverte, 2002, p. 13.

14 Sur la vogue des « revues nègres », voir A. DECORET-AHIHA, Les danses exotiques en France (1880-1940), Pantin, Centre National de la Danse, 2004. 
15 Idem, p. 90.

16 Expression empruntée à l'un des critique de la Revue de Joséphine Baker (André Levinsin, 1929) cité par Blanchard, E. Deroo et G.Manceron, Le Paris Noir, Paris, Editions Hazan, 2001, p. 78. Sur les articulations érotiques des danses exotiques en Europe (et leur succès), voir J-F. STASZAK, Danse exotique, danse érotique. Perspectives géographiques sur la mise en scène du corps de l'Autre (XVIIIème-XXIème siècles), dans Annales de Géographie, vol. 660-661, 2008, t. 2.

17 F. VAn Der Linden, Le Congo, les Noirs et Nous, Paris, Editions Challamel, 1910, p. 108-109.

18 L'explorateur britannique Thomson (1858-1895) est ici cité par J. FABIAN, Out of our minds. Reason and madness in the Exploration of Central Africa, Berkeley, University of California Press, 2000, p. 79.

19 Alphonse Cabra est cité par P. DECAPMAKER, Danses des Bakongo, dans La Mission et les joies populaires. Volksvermaak in de Missie. Compte-rendu de la XVIème semaine de missiologie de Louvain, Paris, L'Édition Universelle ; Bruxelles, Desclée De Brouwer et Cie, 1938, p. 45-46.

20 L. JEURISSEN, Colonisation au masculin et mise en corps de la féminité noire : le cas de l'ancien Congo Belge, (texte de la conférence du jeudi 8 mai 2003 dans le cadre du cycle «Femme et corps »), http://www.ferulg.ulg.ac.be/e_textes.html (consulté le 12 mars 2015).

21 Rapport AT (Administrateur territorial) Bakwa. «La danse des Banda », 28 mai 1933, AA (Archives Africaines, Bruxelles), GG (Fonds du Gouvernement Général), (8285), dossier « Sectes secrètes ».

22 C. VAN De LanoItTE, Sur les rivières glauques de l'Equateur. Trois années en brousse congolaise, Bruxelles, Iris, 1932, p. 188.

23 A. Ruscio, Le credo de l'homme blanc. Regards coloniaux français XIXème-XXème siècles, Bruxelles, Complexe, 1995, p. 188-189.

24 P. DeCAPMAKer, Danses des Bakongo..., p. 43-44.

25 Y. Covington-WARD, Threatening Gestures, Immoral Bodies..., p. 88 et suiv.

$\underline{26}$ Arrêté du 17 juillet 1900 du GG (Gouverneur général) relatif aux danses indigènes, EIC GOUVERNEMENT LOCAL, Recueil mensuel des ordonnances, arrêtés, circulaires, instructions et ordres de service, 1900, p. 95.

27 Voir par exemple les dossiers AA, GG (19542) et AA, GG (6172). Ces interdictions semblent également renforcées en période de trouble, où le caractère anti-européen de certaines performances inquiète particulièrement l'administration coloniale ; ce fut notamment le cas au cours des deux guerres mondiales (voir le dossier AA, GG (6059)). Pour le cas spécifique du Kimbanguisme, voir Covington-WARD, Threatening Gestures, Immoral Bodies..., p. 82 et suiv.

28 Note du Commissaire de district Kwango, 28 janvier 1941. AA, GG (18799), dossier « Divers e.a. danses obscènes ». Ici encore, il faut noter que ces interdictions semblent particulièrement fortes en période de tensions (ici dans le contexte de la Seconde Guerre mondiale, ailleurs dans le contexte de la crise économique du début des années 1930, voir dossier AA, GG (8285), dossier « Polygamie - dot - tutelle - danses »). 
« Notre peuple a perdu le sens de la danse honnête ». Danses africaines, caté...

29 Circulaire interprétative de l'arrêté du GG du 17 juillet 1900 relatif aux danses indigènes, du 16 janvier 1912 du Vice-GG, Congo BeLGe- Gouvernement local. Recueil bi-mensuel des ordonnances, circulaires, instructions et ordres de service, 1912, p. 71.

30 Circulaire relative aux danses indigènes du $1^{\mathrm{er}}$ octobre 1913 par le Vice-GG, ConGo BELGEGOUVERNEMENT LOCAL. Recueil bi-mensuel des ordonnances...,1913, p. 287.

31 Circulaire relative aux danses indigènes du 11 janvier 1915 du GG, CONGO BeLGEGoUvERnEMENT LOCAL. Recueil bi-mensuel des ordonnances..., 1915, p. 8. Pour une description d'ensemble plus détaillée de cette législation, on se reportera utilement à COVINGTON-WARD, Threatening Gestures, Immoral Bodies..., p. 88 et suiv.

32 Mémoire sur la politique indigène et les missions catholiques du Congo Belge, du Secrétaire du Comité permanent des missions catholiques - préfet apostolique du Kwango au GG, 10 octobre 1923, AA, AI (Fonds Affaires Indigènes) (1485).

33 Comme en atteste par exemple le courrier du Vicaire apostolique du Kwango à [illisible], 3 novembre 1930, AA, GG (20702).

34 Voir par exemple des incidents survenus en 1931 dans le Kwango (AA, GG (20702)) et à plusieurs reprises dans les années 1930 dans la province de Léopoldville (AA, GG (21032), dossier « Danses indigènes »).

35 COVInGton-WARD, Threatening Gestures, Immoral Bodies..., p. 88 et suiv.

36 Voir par exemple les recommandations du Commissaire de district assistant du Kwango à tous. AT, 28 juillet 1929, AA GG (19129), dossier AI/C.11 « Danses indigènes ».

37 Note du Commissaire de district Kikwit, 11 avril 1941, GG (18799), dossier « Divers e.a. danses obscènes ».

38 Courrier de AT Bapende à Commissaire de district Kasaï, 15 juin 1935, AA, GG (19116), dossier « Ngobo ».

39 P. DeCAPMAKER, Danses des Bakongo..., p. 49.

40 Courrier de AT Fesho à Commissaire de district Kwango, 19 décembre 1934, AA, GG (19116), dossier « Ngobo ».

41 Chalux, Un an au Congo Belge, Bruxelles, 1925, p. 157.

$\underline{42}$ P. MARTIN, Leisure and Society in Colonial Brazzaville..., p. 132.

$\underline{43}$ C'est en tout cas ce que rapporte Chalux pour les dancings de Kinshasa (CHAlux, Un an au Congo..., p. 156).

44 C.D. Gondola, Villes miroirs..., p. 197.

$\underline{45}$ En effet, «la » danse africaine est souvent présentée comme liée à des buts magiques, spirituels ou religieux, et ainsi opposée à la danse européenne classique, qui serait quant à elle motivée par une recherche esthétique, des représentations qui méritent d'être repensées comme 
le suggère notamment M. LASSIBILE, 'La danse africaine', une catégorie à déconstruire. Une étude des danses des WoDaaBe du Niger, dans Cahiers d'Etudes Africaines, vol. 175, 2004.

46 M. TCheBwa, Terre de la chanson. La musique zaïroise hier et aujourd'hui, Louvain La Neuve, De Boeck Université, 1996, p. 66.

47 B. JEWSIEWICKI, Introduction. De la musique katangaise aux musiques du Katanga, dans Musique urbaine au Katanga. De Malaika à Santu Kimbangu, éd. B. JEWSIEWICKI, Paris, L'Harmattan, 2003, p. 4. Dans les années 1910, certaines performances musicales publiques de l'association Butwa (Elizabethville) témoignent par exemple d'une hostilité certaine envers les Européens, comme l'a montré B. FETTER, The Creation of Elisabethville, 1910-1940, Stanford, Hoover Institution Press, 1976, p. 39.

$48 \mathrm{Au}$ Congo, colonisation belge oblige, la chanson française aura une influence particulièrement marquante. Voir C.D. GondolA, Villes miroirs..., p. 207-208.

$\underline{49}$ Sur ce dernier point, voir notamment G. STEWART, Rumba on the River : A History of the Popular Music of the Two Congos, New York, Verso, 2000, p. 14.

50 C.D. Gondola, Villes miroirs..., p. 204-205.

51 G. STEWART, Rumba on the River..., p. 16.

52 P. MARTIN, Leisure and Society..., p. 131-132.

53 Ibidem.

54 Idem, p. 133-136.

55 G. STEWART, Rumba on the River..., p. 21.

56 La rumba est introduite dans les villes du Congo dans la deuxième moitié des années 1930 ; elle ne prendra l'essor considérable qu'on lui connaît qu'après la Seconde Guerre mondiale, donnant naissance à une "rumba congolaise » célébrée dans toute l'Afrique. Sur l'importance du rôle de la rumba et les raisons de sa popularité, voir B. WHITE, Congolese Rumba and Other Cosmopolitanisms, dans Cahiers d'Etudes Africaines, vol. 168, 2002 et B. WHITE, Rumba Rules....

$\underline{57}$ Note du Procureur général du Roi d’Elisabethville à Gouverneur de province Katanga, 17 octobre 1927, AA, GG (6059), dossier « Danses. Décisions. Instructions ».

58 Courrier du service Population Noire Léopoldville à Commissaire de district Moyen-Congo, 19 juillet 1943, AA, GG (17558).

59 Du Vicaire apostolique de Coquilhatville à AT Coquilhatville, 28 juin 1944, AA, GG (8825).

60 P. MARTin, Leisure and Society..., p. 132. On retrouve également cette dimension dans d'autres métropoles d'Afrique sub-saharienne, voir par exemple J. PRAIS, Representing an African City and Urban Elite : The Nightclubs, Dance Halls, and Red-Light District of Interwar Accra, dans The Arts of Citizenship in Africa Cities, éd. R. Fredericks ET M. Diouf, New York, Palgrave, 2014.

61 B. JEWSIEWICKI, Introduction. De la musique katangaise..., p. 4. Sur ce sujet, voir aussi B. 
« Notre peuple a perdu le sens de la danse honnête ». Danses africaines, caté...

JEWSIEWICKI, Danse et construction de l'identité de la personne moderne en Afrique centrale..., p. 101-110 et Dance : The Passion to Be Oneself..., p. 171-183.

62 Comme le note Phyllis Martin, « certainly, the elite tended to dance to the European and American music and older people might prefer the older dance form, but there were many who liked both. Two men who danced with their regional associations on Sunday afternoon said that they went to 'bars-dancings' in the evenings ». P. MARTIN, Leisure and Society..., p. 151-152.

63 Courrier du service Population Noire Léopoldville à Commissaire de district Moyen-Congo, 19 juillet 1943, AA, GG (17558).

64 A. Gillès DE PELICHY, Les réjouissances populaires au Katanga, dans La Mission et les joies populaires..., p. 149.

$\underline{65}$ Courrier de AT Kikwit à Commissaire de district Kwango, 6 août 1931, AA, GG (20702).

$\underline{66}$ Note «Salles de danses » du Chef de camps de la cité indigène d'Élisabethville, 3 juin 1928, AA, GG (6059), dossier « Danses. Décisions. Instructions ».

$\underline{67}$ Courrier de Commissaire de district Elisabethville à AT Élisabethville, 11 mars 1933, AA, GG (6059), dossier « Danses. Décisions. Instructions ».

68 Compte rendu analytique de la réunion du Comité Protecteur des centres extracoutumiers du Katanga, 15 février 1933, AA, GG (16419), dossier « Centres extracoutumiers ».

$\underline{69}$ «Bien des faiblesses ont été exploitées par des tenanciers étrangers sans foi ni loi, qui y ont trouvé leur profit. Des salles de danse européennes et des débits de boisson ont été ouverts un peu partout. Les Noirs ont pris goût à la Maringa et à d'autres danses fort légères. La bière et les liqueurs européennes, plus fortes que la plupart des boissons indigènes, ont été bien vite appréciées, et la moralité des centres extracoutumiers a baissé dans des proportions inquiétantes. » A. Gillès DE PELICHY, Les réjouissances populaires au Katanga ..., p. 138.

$\underline{70}$ « Note au sujet des faits relevés dans la lettre du 30 mars adressée à Monsieur le Gouverneur de la province par Monseigneur de Hemptinne et Monsieur le docteur Mottoulle », du commissaire de district Élisabethville, 5 avril 1933, AA, GG 6059, dossier « Danses. Décisions. Instructions ».

\section{Ibidem.}

72 Le Bulletin de la Ligue Nationale de Moralité Publique (1951) est cité par L. Di SpURIO, Le temps de l'amour. Jeunesse et sexualité en Belgique francophone (1945-1968), Bruxelles, Le Cri, p. 117. Sur le sujet, voir aussi Idem, p. 116-126 et Dancings. Le marché aux filles, dans L'Heure Bleue. La vie nocturne à Bruxelles de 1830 à 1940, Bruxelles, Crédit Communal, 1987, p. 137-143.

73 Lettre-circulaire de Chef de province Léopoldville à tous Commissaires district et AT, 25 septembre 1936, AA, GG (21032), dossier « Danses indigènes ».

$\underline{74}$ Du Substitut du procureur du roi d'Élisabethville à Gouverneur de province, 9 mars 1928, AA, GG (6059), dossier « Danses. Décisions. Instructions ».

$\underline{75}$ Ordonnance $n^{\circ}$ 245/J du GG (Congo-Kasaï) du 19 décembre 1931, ordonnance n 18 du 
Gouverneur du Katanga, 16 décembre 1932, ordonnance n 94/AIMO du gouverneur de l'Équateur, 13 juin 1933, dans P. PIRON et J. DE Vos, Codes et Lois du Congo Belge, Bruxelles/ Léopoldville, Larcier/Editions des Codes et Lois du Congo Belge, 1954, p. 585-586.

$\underline{76}$ En matière de ségrégation, les mesures interdisent aux Européens d'ouvrir des dancings dans les circonscriptions « indigènes » et aux Africains d'ouvrir des dancings dans les circonscriptions européennes; de plus, le simple accès des dancings installés dans les quartiers européens à des personnes « de race indigène » est prohibé. En matière d'ouverture et de fonctionnement, les mesures imposent une autorisation préalable du commissaire de district et une licence (pour les propriétaires « non-indigènes » principalement).

77 Qui sera aussi complété par des dispositions techniques et de sécurité. Voir principalement l'ordonnance $\mathrm{n}^{\circ}$ 328/TP du 28 octobre 1944 et ordonnance ${ }^{\circ}$ 348/AIMO du 29 octobre 1947, dans P. Piron et J. De Vos, Codes et Lois du Congo Belge..., p. 1543-1547 et 876-877. Cette dernière réglemente également les autorisations de danse de manière plus large.

78 Rapport annuel 1930 et 1931 des cités indigènes du district urbain de Léopoldville, AA, RA/ CB, AIMO (119) et (120).

79 Courrier du service Population Noire Léopoldville à Commissaire de district Moyen-Congo, 19 juillet 1943, AA, GG (17558).

80 Notamment en interdisant de délivrer des licences d'exploitation pour : des commerçants disposant déjà d'une licence de vente des boissons alcooliques, des locaux situés à proximité d'un débit de boissons; des personnes ayant un casier judiciaire ou « dont la conduite ou celle des personnes vivant sous le même toit (...) est répréhensible au point de vue moral », Projet de décision du Commissaire de district Élisabethville, 2 octobre 1927, AA, GG (6059), dossier «Danses. Décisions. Instructions ».

81 Décision du Commissaire de district d'Elisabethville, 30 avril 1931, AA, GG (6059), dossier « Danses. Décisions. Instructions ».

\section{Ibidem.}

83 Compte rendu analytique de la réunion du Comité Protecteur des centres extracoutumiers du Katanga, 15 février 1933, AA, GG (16419), dossier « Centres extracoutumiers ».

84 Courrier de Commissaire de district Élisabethville à AT Elisabethville, 11 mars 1933, AA, GG (6059), dossier « Danses. Décisions. Instructions ».

85 En vertu de l'Arrêté $\mathrm{n}^{\circ} 14$ du 25 avril 1934. En 1936 déjà, plusieurs salles de danse plus ou moins clandestines avaient ré-ouvert. Courrier de Commissaire de district Élisabethville à AT Élisabethville, 9 décembre 1936, AA, GG (6059), dossier « Danses. Décisions. Instructions ».

86 C.D. Gondola, 'Bisengo ya la joie'..., p. 95.

87 Pour un exemple illustratif de l'intérêt de ce type d'approche, voir E. CALLACI, DanceHall Politics : Mobility, Sexuality, and Spectacles of Racial Respectability in Late Colonial Tanganyika 1930s-1961, dans Journal of African History, vol. 52, 2011, t. 3. Dans le cas du Congo, la figure de l'urbain « de petite vertu » fréquentant les bars et les dancings est établie comme un repoussoir 
« Notre peuple a perdu le sens de la danse honnête ». Danses africaines, caté...

pour les «Evolués », dans les colonnes de La Voix du Congolais par exemple (voir D. Todt, «Les Noirs perfectionnés ». Cultural Embourgeoisement in Belgian Congo during the 1940s and 1950s, Sonderforschungsbereich 640 : Repräsentationen sozialer Ordnungen im Wandel 4 (SFB 640 Papers), Berlin, 2012, p. 13), même si ce n'est pas le moindre des paradoxes du monde de la fête kinois que de faire «se rencontrer l'évolué, l'avenir du colonisateur, et la ndumba, sa hantise » (C.D. GondolA, 'Bisengo ya la joie'..., p. 95).

88 Deux excellentes études ont récemment interrogé les discours sur la « moralité » (profane ou sacrée) de certaines danses : L.N. BRAun, Trading Virtue for Virtuosity. The Artistry of Kinshasa's Concert Danseuses, dans African Arts, vol. 47, 2014, 4. Voir également K. PYPE, Dancing for God or the Devil : Pentecotalist Discourse on Popular Dance in Kinshasa, dans Journal of Religion in Africa, vol. 36, 2006, 3/4.

89 Je reprends ici le titre d'un des chapitres de F. RAMIREZ et C. ROLOT, Histoire du cinéma colonial au Zaïre, au Rwanda et au Burundi, Tervuren, Musée Royal de l’Afrique Centrale, 1985.

90 J. VAN WING, Les danses Bakongo, p. 130.

91 Intervention du RP F. AUPIAIs faisant suite à P. DECAPMAKER, Danses des Bakongo..., p. 58.

92 Annexe au courrier du Conseil d'administration de l'ASBL « Ndako Ya Bisu. Notre maison- Ons huis » au Ministre des colonies, 29 septembre 1931, AA, AI (1396), liasse «6.a. Euvres sociales ».

93 F. RAMirez et C. Rolot, Histoire du cinéma colonial ..., p. 55.

94 Comme en témoigne par exemple le film A travers l’Ubangi de G-J. Felix, 1949, 15 min.

95 Voir notamment G. VANTHEMsche, Genèse et portée du 'Plan Décennal' au Congo Belge, Bruxelles, ARSOM, 1994.

$\underline{96}$ Note confidentielle du Vice-GG (pour le GG) à Gouverneur province Léopoldville, 31 décembre 1951, AA, GG (5950).

$\underline{97}$ Sur la réception de Bwana Kitoko par les spectateurs congolais, voir M.Z. AzIZA ETAMBALA, «Bwana Kitoko » (1955), un film d'André Cauvin : réalités congolaises ou rêveries belges, dans Patrimoine filmé d'Afrique centrale. Archives films. Congo, Rwanda, Burundi, 1912-1960, eds. P. VAn Schuylenbergh et M.Z. Aziza Etambala, Tervuren, Musée Royal de l’Afrique Centrale, 2010.

PDF généré automatiquement le 2020-07-07 20:41:25

Url de l'article : https://popups.uliege.be:443/1370-2262/index.php?id=246 